\title{
AKARI's infrared view on nearby stars
}

\section{Using AKARI infrared camera all-sky survey, 2MASS, and Hipparcos catalogs}

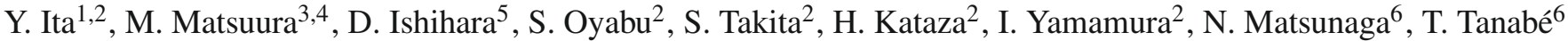 \\ Y. Nakada ${ }^{6}$, H. Fujiwara ${ }^{7}$, T. Wada ${ }^{2}$, T. Onaka ${ }^{7}$, and H. Matsuhara ${ }^{2}$ \\ 1 National Astronomical Observatory of Japan, 2-21-1 Osawa, Mitaka, Tokyo, 181-8588, Japan \\ e-mail: yoshifusa.ita@nao.ac.jp; yita@ir.isas.jaxa.jp \\ 2 Institute of Space and Astronautical Science, Japan Aerospace Exploration Agency, 3-1-1 Yoshinodai, Sagamihara, \\ Kanagawa 229-8510, Japan \\ 3 UCL-Institute of Origins, Department of Physics and Astronomy, University College London Gower Street, London WC1E 6BT, \\ $\mathrm{UK}$ \\ ${ }^{4}$ UCL-Institute of Origins, Mullard Space Science Laboratory, University College London, Holmbury St. Mary, Dorking, \\ Surrey RH5 6NT, UK \\ 5 Department of Astrophysics, Nagoya University, Chikusa-ku, Nagoya 464-8602, Japan \\ 6 Institute of Astronomy, School of Science, The University of Tokyo, Mitaka, Tokyo 181-0015, Japan \\ 7 Department of Astronomy, Graduate School of Science, The University of Tokyo, Bunkyo-ku, Tokyo 113-0033, Japan
}

Received 19 November 2009 / Accepted 27 February 2010

\section{ABSTRACT}

\begin{abstract}
Context. The AKARI, a Japanese infrared space mission, has performed an All-Sky Survey in six infrared-bands from 9 to $180 \mu \mathrm{m}$ with higher spatial resolutions and better sensitivities than IRAS.

Aims. We investigate the mid-infrared (9 and $18 \mu \mathrm{m}$ ) point source catalog (PSC) obtained with the infrared camera (IRC) onboard AKARI, in order to understand the infrared nature of the known objects and to identify previously unknown objects.

Methods. Color-color diagrams and a color-magnitude diagram were plotted with the AKARI-IRC PSC and other available all-sky survey catalogs. We combined the Hipparcos astrometric catalog and the 2MASS all-sky survey catalog with the AKARI-IRC PSC. We furthermore searched literature and SIMBAD astronomical database for object types, spectral types, and luminosity classes. We identified the locations of representative stars and objects on the color-magnitude and color-color diagram schemes. The properties of unclassified sources can be inferred from their locations on these diagrams.

Results. We found that the $(B-V)$ vs. $(V-S 9 W)$ color-color diagram is useful for identifying the stars with infrared excess emerged from circumstellar envelopes or disks. Be stars with infrared excess are separated well from other types of stars in this diagram. Whereas $(J-L 18 W)$ vs. ( $S 9 W-L 18 W)$ diagram is a powerful tool for classifying several object types. Carbon-rich asymptotic giant branch (AGB) stars and OH/IR stars form distinct sequences in this color-color diagram. Young stellar objects (YSOs), pre-main sequence (PMS) stars, post-AGB stars, and planetary nebulae (PNe) have the largest mid-infrared color excess and can be identified in the infrared catalog. Finally, we plot the $L 18 W$ vs. $(S 9 W-L 18 W)$ color-magnitude diagram, using the AKARI data together with Hipparcos parallaxes. This diagram can be used to identify low-mass YSOs and AGB stars. We found that this diagram is comparable to the [24] vs. ([8.0] - [24]) diagram of Large Magellanic Cloud sources using the Spitzer Space Telescope data. Our understanding of Galactic objects will be used to interpret color-magnitude diagram of stellar populations in the nearby galaxies that Spitzer Space Telescope observed.

Conclusions. Our study of the AKARI color-color and color-magnitude diagrams will be used to explore properties of unknown objects in the future. In addition, our analysis highlights a future key project to understand stellar evolution with a circumstellar envelope, once the forthcoming astronometrical data with GAIA are available.
\end{abstract}

Key words. stars: AGB and post-AGB - stars: emission-line, Be - supergiants - stars: Wolf-Rayet - stars: pre-main sequence infrared: stars

\section{Introduction}

More than a quarter century passed since the pioneering infrared whole-sky survey of IRAS, which covered more than $96 \%$ of the whole-sky in four photometric bands at $12,25,60$, and $100 \mu \mathrm{m}$

* Catalogs (full Tables 3 and 4) are available in electronic form at the CDS via anonymous ftp to

cdsarc.u-strasbg.fr $(130.79 .128 .5)$ or via

http://cdsweb.u-strasbg.fr/cgi-bin/qcat?J/A+A/514/A2
(IRAS Explanatory Supplement 1988). The IRAS point source catalog (PSC) has shown that mid- and far-infrared census is essential for studying dust embedded objects, such as star-forming regions, debris disks around main sequence stars, evolved stars, and distant galaxies. However, the spatial resolution was not as good for studying sources in the crowded regions. After the IRAS, the COsmic Background Explorer (DIRBE/COBE; Hauser et al. 1998) has mapped the whole-sky in 1.25-240 $\mu \mathrm{m}$ 
with 10 photometric-bands. It intended to accurately obtain the intensity of diffuse background radiation and did not have high enough sensitivity for point sources.

In the meantime, ultra-violet, optical, and near-infrared large area surveys have been carried out using ground-based telescopes (e.g. GALEX, Martin et al. 2005; SDSS, York et al. 2000; and 2MASS, Skrutskie et al. 2006). Their counterparts are missing or hard to be uniquely identified in mid-infrared and farinfrared catalogs, which prevents us from studying objects surrounded by dust. The demand for a new mid- and far-infrared whole-sky survey with better sensitivity and higher spatial resolution has increased. To fulfill these expectations, AKARI, a Japanese infrared satellite was launched at 21:28 UTC on February 21, 2006 from the Uchinoura Space Center (Murakami et al. 2007). Sharing the time with pointed observations, AKARI has mapped the whole-sky in mid- and far-infrared using two instruments onboard; the infrared camera (IRC; Onaka et al. 2007) and the far-infrared surveyor (FIS; Kawada et al. 2007). The FIS swept about $94 \%$ of the whole-sky more than twice at $65,90,140$, and $160 \mu \mathrm{m}$ wavebands. Also, the IRC swept more than $90 \%$ of the whole-sky more than twice using two filter bands centered at $9(S 9 W, 7-12 \mu \mathrm{m})$ and $18(L 18 W, 14-25 \mu \mathrm{m})$ $\mu \mathrm{m}$ (Ishihara et al. 2010). These abbreviated filter band names are used throughout this article. The cut-in and cut-off wavelengths indicated in parenthesis correspond to those where the throughput becomes a half of the peak. See Fig. 1 for the normalized spectral response function of the IRC bands.

In this paper, we use the IRC mid-infrared all-sky survey data to study Galactic stellar objects. Compared to the IRAS survey, the sensitivities at 9 and $18 \mu \mathrm{m}$ bands are more than 15 and 5 times better than those of the IRAS's 12 and $24 \mu \mathrm{m}$ bands, and the spatial resolution is more than 100 times finer for the IRC survey. Van der Veen \& Habing (1988) utilized IRAS mid- and far-infrared combined colors to diagnose the nature of IRAS sources. They defined color criteria to classify sources into several groups, and since then those criteria have been used by many authors. Taking this as a role model, we crossidentified the AKARI IRC all-sky survey point source catalog with the Hipparcos astrometric catalog (van Leeuwen 2007) and the 2MASS all-sky survey catalog (Skrutskie et al. 2006) to classify sources on color-color and color-magnitude diagrams. The main objective of this paper is to study overall infrared characteristics of galactic stellar sources. The new all-sky survey catalog should be useful for wide variety of astronomical studies. Practical applications of the catalog are to search for hot debris disk (Fujiwara et al. 2010), extragalactic objects (Oyabu et al., in preparation) and to study YSOs (Takita et al. 2010).

In the next section we show the general characteristics of the AKARI IRC all-sky survey point source catalog, but also see Ishihara et al. (2010) for the complete description of the AllSky Survey, its data reduction processes, the point source catalog compilation processes, and the catalog characteristics.

\section{Characteristics of the AKARI IRC all-sky survey catalog}

The first release version (ver. $\beta$-1) of the AKARI IRC midinfrared all-sky survey point source catalog (IRC-PSC) lists more than 851000 and 195000 sources in the 9 and $18 \mu \mathrm{m}$ bands, respectively. There are about 170000 sources detected in the two bands. This number is in a range expected from the difference in the detection limit between the two bands and the fall of the Rayleigh-Jeans spectrum since most of the sources are

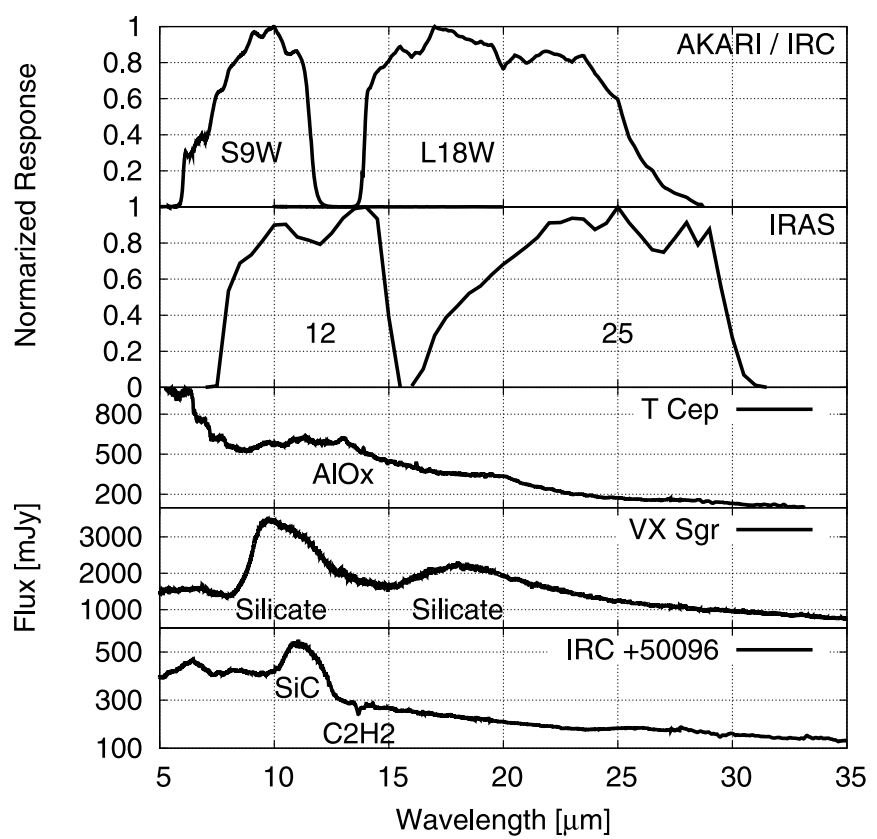

Fig. 1. The normalized spectral response function of the AKARI IRC bands and the IRAS bands. As references, the ISO SWS spectra of three representative Galactic AGB stars (T Cep as O-rich AGB with AlOx feature, VX Sgr as O-rich AGB with silicate feature, and IRC+50096 as $\mathrm{C}$-rich $\mathrm{AGB}$ with $\mathrm{SiC}$ feature) with circumstellar dust features are also shown.

stars. The estimated $5 \sigma$ detection limits for one scan are about 50 and $90 \mathrm{mJy}$ in the 9 and $18 \mu \mathrm{m}$ bands, respectively. The present catalog includes point-like sources that were detected more than twice. The sensitivity will be improved in the future catalog for sources in high visibility regions for AKARI's sun-synchronous orbit (i.e., high ecliptic latitude regions), where AKARI scanned many times. The saturation limits depend on the sky region, and the brightest source listed in the catalog is about 560 and $1200 \mathrm{Jy}$ in the 9 and $18 \mu \mathrm{m}$ bands, respectively. The pixel field of view of the survey observation mode is about $10 \mathrm{arcsec}$, and the positional accuracy of detected sources is better than 3 arcsec.

\subsection{Flux accuracy}

\subsubsection{Comparison to the IRAS point source catalog}

Although the band profiles of AKARI IRC's $S 9 W$ and $L 18 \mathrm{~W}$ bands and IRAS's 12 and $25 \mu \mathrm{m}$ bands are different, a comparison of the photometry of common sources is useful for testing the calibration of the IRC-PSC. In Fig. 1 we show the normalized spectral response function of the AKARI and the IRAS ${ }^{1}$ bands. The ISO SWS spectra (Sloan et al. 2003a) of some galactic stars with characteristic circumstellar dust features are also shown to give a rough idea of the cause of differences in photometry between the associated filter bands.

The IRAS-PSC lists 245888 sources, among which 170754 have a flux quality flag better than 1 in at least one of the 12 and $25 \mu \mathrm{m}$ bands (i.e., $f_{q 12}>1$ or $f_{q 25}>1$ ). We consider these 170754 sources as good IRAS sources and find AKARI counterparts for $145751(>85 \%)$ good IRAS sources using the simple positional matching method with a tolerance radius of 30 arcsec.

\footnotetext{
1 Data taken from

http://irsa.ipac.caltech.edu/IRASdocs/exp.sup/ch2/ tabC5.html
} 


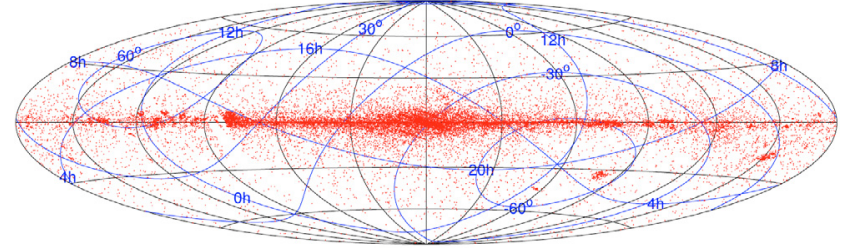

IRAS sources without AKARI detection

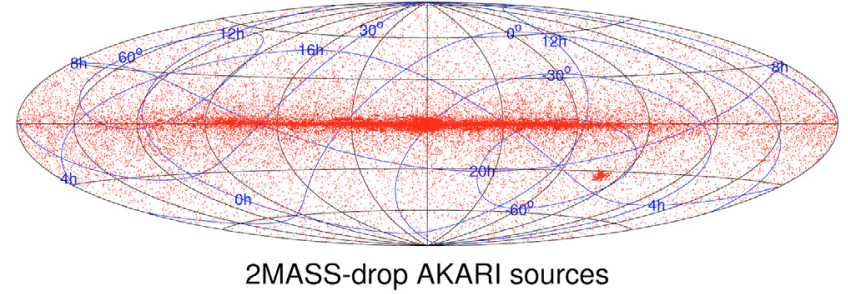

Fig. 2. A projection of the IRAS sources without AKARI detection (upper panel) and the 2MASS-drop AKARI sources (lower panel) onto the galactic coordinate map. The blue lines show the equatorial coordinate.

In some cases, more than one AKARI point source are found for a single IRAS source. In these cases, we only adopt the closest one and regard the other(s) as unmatched, even if they are actually multiple sources resolved by AKARI that appear as one source to the IRAS. We compared the AKARI and IRAS photometry of the matched sources to find that the photometry in the IRC-PSC and the IRAS-PSC agrees within 37 and $40 \%$ in $S 9 W$ vs. IRAS 12 and $L 18 W$ vs. IRAS 25 for sources with an IRAS flux quality flag of 3 . If we compare a subsample of high galactic latitude $\left(|b|>30^{\circ}\right)$ and high-quality $(S / N>10$ in IRC bands) sources, their photometry are in agreement within 18 in $S 9 W$ vs. IRAS 12 and $24 \%$ in $L 18 W$ vs. IRAS 25 (see also Ishihara et al., 2010). The comparison also reveals that there are about 25000 good IRAS sources that have no AKARI counterparts. Their galactic spatial distribution is shown in the upper panel of Fig. 2. There are several possibilities why some of the IRAS sources (especially bright ones) are not listed in the IRCPSC: (1) they are saturated in the AKARI survey; (2) the IRAS point-like source can be recognized as an extended source to the AKARI's eye, so is excluded from the "point source" catalog; (3) the IRAS source can be resolved into several fainter sources to the AKARI's eye; (4) there is a difference in the sky coverage ( $\sim 90 \%$ for AKARI and $\sim 96 \%$ for IRAS) or it is located in the unexplored sky region (Ishihara et al. 2010).

\subsection{Positional accuracy}

The 2MASS point source catalog (2MASS-PSC; Skrutskie et al. 2006) is complete down to $K_{\mathrm{s}}<14.3 \mathrm{mag}$ in the absence of confusion $^{2}$. Then, all normal stars seen by AKARI should be prominent in the 2MASS-PSC. Therefore, we used the 2MASSPSC to assess the positional accuracy of the IRC-PSC.

There are 761565 sources with $S / N>5$ at the $S 9 W$ band in the IRC-PSC. We consider these 761565 sources to be good IRC sources. We searched for the 2MASS counterparts brighter than $14.3 \mathrm{mag}$ and $S / N>5$ in the $K_{\mathrm{s}}$ band for the good IRC sources. If more than one $2 \mathrm{MASS}$ source are found within the tolerance radius from an AKARI source, we only adopted the closest one and regarded the other(s) as unmatched. We find

\footnotetext{
2 http://www.ipac. caltech.edu/2mass/releases/allsky/ doc/sec2_2.html
}

counterparts for 505350 (66\%), 673730 (88\%), 713705 (94\%), $724739(95 \%)$, and $728178(96 \%)$ good IRC sources using the positional tolerance radii of $1-5$ arcsec, respectively. If we use a subsample of 562598 high quality $(S / N>10$ at the $S 9 W$ band) IRC-PSC sources, the results would be $68 \%, 88 \%, 93 \%, 94 \%$, and $94 \%$ for the positional tolerance radii of $1-5$ arcsec, respectively. These results suggest that the positional accuracy of the IRC-PSC does not depend on the source brightness, and the accuracy is uniform at least for sources with $S / N>5$. Also, it seems that the chance for false matches may increase if we use the tolerance radius larger than 4 arcsec.

To see the dependence of positional accuracy on the source density, we made the same 2MASS counterpart search with a subsample of 78171 high galactic latitude $\left(|b|>30^{\circ}\right)$ good IRC-PSC sources, where we do not suffer from severe confusion. We searched for the 2MASS counterpart brighter than $14.3 \mathrm{mag}$ and $S / N>5$ in $K_{\mathrm{S}}$ band for these high-latitude, good IRC-PSC sources, and find counterpart for $61619(79 \%)$, 70446 (90\%), 71877 (92\%), 72334 (93\%), and 72528 (93\%) sources using the positional tolerance radii of $1-5$ arcsec, respectively. This result indicates that the positional accuracy of the IRC-PSC may depend on the source density, but it does not matter if we use a match radius larger than 2 arcsec.

Considering the above test results, we conclude that the positional accuracy of the IRC-PSC sources is better than 3 arcsec for most of the sources. This result is compatible with the pointing accuracy estimated in Ishihara et al. (2010). Then we decided to use a tolerance radius of 3 arcsec for the catalog comparisons in the next section.

Finally, the AKARI sources without 2MASS counterpart should be of particular interest because such sources can be deeply dust enshrouded objects (e.g., OH/IR stars, dusty carbon stars, etc.) or distant galaxies. There are 47860 good IRC sources without 2MASS counterpart (brighter than $14.3 \mathrm{mag}$ and $S / N>5$ in $K_{\mathrm{s}}$ band) within a radius of 3 arcsec. We show the galactic spatial distribution of these 2MASS-drop AKARI sources in the lower panel of the Fig. 2. Follow-up observations are definitely needed to identify these sources.

\section{Cross-identification with existing catalogs/databases and data set definition}

The IRC-PSC is cross-identified with existing all-sky survey catalogs, namely the new Hipparcos astrometric catalog (van Leeuwen 2007) and the 2MASS PSC (Skrutskie et al. 2006), using a simple positional correlation method. The epoch of the source positions listed in the new Hipparcos catalog is 1991.25, while that of the IRC-PSC is 2000.0. There are 15052 Hipparcos sources whose total proper motions are more than 100 milliarcsec/year. Their positions should be corrected for the proper motion over the 8.75 year interval. We calculated the positions in epoch 2000.0 for the Hipparcos sources with good proper motion measurements $\left(\sigma \mu_{\alpha} / \mu_{\alpha}<0.2\right.$ and $\sigma \mu_{\delta} / \mu_{\delta}<0.2$, where $\mu_{\alpha}$ and $\mu_{\delta}$ are proper motions in right ascension and declination, respectively). Then we used the new positions for the cross-identification.

We used a positional tolerance of 3 arcsec amid the positional accuracy of the IRC-PSC $(<3$ arcsec). If more than one source are present within the tolerance radius, the closest one is selected. In Fig. 3, we show the histogram of positional differences for matched sources. We found 68744 matches between the IRC-PSC and new Hipparcos catalog, and 847838 matches between the IRC-PSC and the 2MASS catalog within 


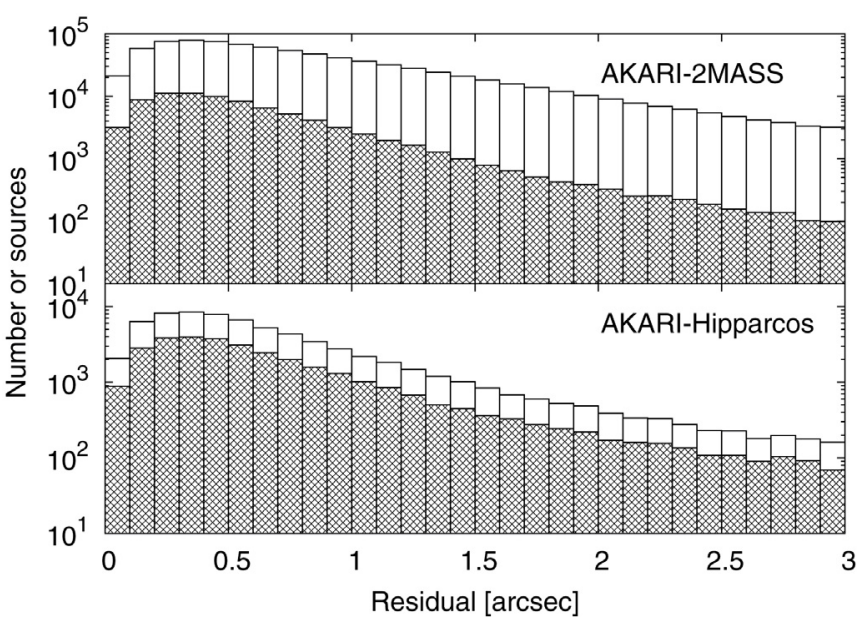

Fig. 3. The histogram of positional differences between the AKARI IRC PSC and the 2MASS catalog (upper panel), and the Hipparcos catalog (lower panel) for matched sources. The hatched areas represent matched sources in the high galactic latitude $\left(|b|>30^{\circ}\right)$ region. The size of the bin is 0.1 arcsec.

the tolerance radius of 3 arcsec. The optical and near-infrared photometries used in this article were taken from these catalogs. Magnitudes were not corrected for interstellar extinction. Instead, we indicated interstellar extinction by showing extinction vector. We used the Weingartner \& Draine (2001) extinction model for the Milky Way of $R_{v}=3.1$ to calculate extinction vectors. We took improved astrometry, $(B-V)$ colors and their errors, and $(V-I)$ colors from van Leeuwen (2007), and $V$-band magnitudes were extracted from the older version of the Hipparcos catalog (ESA, 1997). We assumed that the errors in $V$ magnitudes are $\sigma(V)=\sigma(B-V) / \sqrt{2}$.

\subsection{Sources with known classifications}

After the cross-identification processes, we searched astronomical catalogs that compile interesting types of objects. Then we cross-identified our data with the following astronomical catalogs using a tolerance radius of 3 arcsec: (1) S-type stars, i.e., stars with surface carbon-to-oxygen number ratio $(\mathrm{C} / \mathrm{O})$ close to unity: Stephenson (1984, 1990), who list 1412 sources; (2) post-AGB stars: Szczerba et al. (2007), who list 326 very likely post-AGB stars; (3) planetary nebulae: Acker et al. (1994), who list 1143 sources; (4) Wolf-Rayet stars: van der Hucht (2001), who list 226 sources; (5) Be stars: Zhang et al. (2005), who list 1185 sources. We further searched the SIMBAD database for known carbon stars, OH/IR stars, PMS stars, and YSOs, by querying by object types of " $\mathrm{C}^{*}$ ", "OH*", "pMS*", and "Y*O", respectively. Also, M-type giants and supergiants are extracted from the SIMBAD database by querying by spectral types of "M" and luminosity class of "III (giants)" and "I (supergiants)", respectively. We refer to the classifications in the above-mentioned literature and the SIMBAD database query results throughout this article unless otherwise noted. Occasionally, more than one object type are found (within a search radius of 3 arcsec) for a single object in the SIMBAD database. In those cases, we keep both of types.

As for the SIMBAD query results, we eliminated nine sources from the PMS stars list and one source (HD 217086) from the YSOs list. The nine PMS stars were eliminated because these stars are classified as PMS stars by citing Li \& Hu (1998), but there is only one star (HD 21051) among the nine
Table 1. Sources eliminated from the SIMBAD's pre-main-sequence list.

\begin{tabular}{llr}
\hline \hline Name & Adopted object type & Reference \\
\hline VY Ari & Late type stars in binary system & 1 \\
V573 Per & G8 subgiant & 2 \\
HD 21051 & K0 giant & 2 \\
UX Ari & Late type stars in binary system & 3 \\
46 Per & Emission line OB stars & 4 \\
V491 Per & G8 subgiant & 2 \\
V582 Per & F7 dwarf & 5 \\
V492 Per & K1 giant in binary system & 6 \\
111 Tau & F8 dwarf & 7 \\
HD 217086 & O7 dwarf & 8 \\
\hline
\end{tabular}

References. 1: Biazzo et al. (2006); 2: De Mediros \& Mayor (1999); 3: Rosario et al. (2007); 4: Puls et al. (2006); 5: Busa et al. (2007), 6: De Medeiros et al. (2002); 7: Cenarro et al. (2007); 8: Mokiem et al. (2005).

Table 2. Number of sources in our data set with known object types or spectral types.

\begin{tabular}{lrr}
\hline \hline Object types / & \multicolumn{2}{c}{ Data set } \\
Spectral types & 449 & 641 \\
\hline Be stars & 281 & 4606 \\
Carbon stars & 74 & 229 \\
M-type supergiants & 1732 & 3129 \\
M-type giants & 1 & 570 \\
OH/IR stars & 7 & 275 \\
Planetary Nebulae & 42 & 242 \\
Post-AGB stars & 74 & 1229 \\
S-type stars & 54 & 100 \\
Wolf-Rayet stars & 8 & 788 \\
Young stellar objects & 65 & 351 \\
Pre-main-sequence stars & & \\
\hline
\end{tabular}

sources that $\mathrm{Li} \& \mathrm{Hu}$ (1998) indeed identified as a "candidate" weak-lined T Tauri star. The other eight sources are just listed in their paper as possible optical counterparts to the ROSAT all-sky survey bright source catalog (Voges et al. 1996). Also, a literature search made us conclude that HD 217086 is an 07 dwarf, not a YSO. These ten sources are tabulated in Table 1 with their names, adopted object types, and references. After this manual procedure, the number of sources in our data set with known object types/spectral types are summarized in Table 2 . The electronic tables for these data sets are available at the CDS. Tables 3 and 4 are the examples of the data sets provided, and show the first 3 records of them.

\subsubsection{Extragalactic objects}

Extragalactic objects can be contaminants when studying galactic objects in the infrared. As of January 2010, the NASA extragalactic database (NED) lists 2907 classified extragalactic objects with the IRAS $12 \mu \mathrm{m}$ flux brighter than $100 \mathrm{mJy}$. We matched these NED objects with the IRC-PSC and find 794 matches within a tolerance radius of 3 arcsec. This matching result indicates that most of the bright extragalactic objects are identified as an extended source by AKARI, and only distant ones that appear point-like are included in the IRC-PSC. These 794 objects were eliminated from the following analyses. We further investigated contaminant of extragalactic objects in high Galactic latiude regions. There are 85965 AKARI sources detected at either of the $S 9 W$ or $L 18 W$ bands in the region of 
Table 3. The first three records of AKARI-Hipparcos samples with known object types, with sources sorted by increasing order of RA for each object type.

\begin{tabular}{|c|c|c|c|c|c|c|c|c|c|c|c|c|}
\hline \multicolumn{2}{|c|}{$\begin{array}{l}\text { AKARI coordinate } \\
\text { RA and Dec [degree] }\end{array}$} & \multicolumn{3}{|c|}{ [vega-mag] } & \multirow{2}{*}{$\begin{array}{l}\mathrm{e} L 18 W \\
99.999\end{array}$} & \multirow{2}{*}{$\begin{array}{r}\text { Hipparcos } \\
\text { ID } \\
278\end{array}$} & \multicolumn{2}{|c|}{$\begin{array}{c}\text { plx eplx } \\
{[\text { mas }]}\end{array}$} & \multirow{2}{*}{$\frac{V}{940}$} & \multicolumn{2}{|c|}{$\begin{array}{l}(B-V) \quad \mathrm{e}(B-V) \\
\text { [vega-mag] }\end{array}$} & \multirow{2}{*}{$\begin{array}{r}\text { Object } \\
\text { Type } \\
\mathrm{Be}\end{array}$} \\
\hline 0.86307 & 55.55097 & 5.733 & 0.037 & 99.999 & & & 1.57 & 0.82 & & -0.023 & 0.003 & \\
\hline 1.61056 & 64.19614 & 5.424 & 0.026 & 99.999 & 99.999 & 531 & 3.76 & 0.21 & 5.570 & -0.023 & 0.003 & $\mathrm{Be}$ \\
\hline 2.90472 & 58.21186 & 5.457 & 0.024 & 4.592 & 0.103 & 940 & 0.94 & 0.51 & 7.090 & 0.137 & 0.009 & $\mathrm{Be}$ \\
\hline
\end{tabular}

Notes. Any indices with prefix "e" mean their errors. The full version of the table is available at the CDS.

Table 4. The first three records of AKARI-2MASS samples with known object types, with sources sorted by increasing order of RA for each object type.

\begin{tabular}{|c|c|c|c|c|c|c|c|c|c|c|c|c|}
\hline \multicolumn{2}{|c|}{$\begin{array}{l}\text { AKARI coordinate } \\
\text { RA and Dec [degree] }\end{array}$} & $S 9 W$ & $\mathrm{e} S 9 W$ & $L 18 W$ & $\mathrm{e} L 18 W$ & \multicolumn{2}{|c|}{ [vega-mag] } & $H$ & $\mathrm{e} H$ & $K_{\mathrm{s}}$ & $\mathrm{e} K_{\mathrm{s}}$ & $\begin{array}{r}\text { Object } \\
\text { Type }\end{array}$ \\
\hline 0.35326 & 63.50464 & 7.367 & 0.157 & 99.999 & 99.999 & 99.999 & 99.999 & 99.999 & 99.999 & 7.978 & 0.016 & $\mathrm{Be}$ \\
\hline 0.86307 & 097 & 5.733 & 0.037 & 99.999 & 99.999 & 7.266 & 0.018 & 7.225 & 0.046 & 7.090 & 0.020 & $\mathrm{Be}$ \\
\hline 1.61056 & 64.19614 & 5.424 & 0.026 & 99.999 & 99.999 & 5.463 & 0.037 & 5.470 & 0.044 & 5.468 & 0.023 & $\mathrm{Be}$ \\
\hline
\end{tabular}

Notes. Any indices with prefix "e" mean their errors. The full version of the table is available at the CDS.

$|b|>30^{\circ}$. We searched the NED database for galaxies or QSOs within a radius of 3 arcsec from those AKARI sources and obtained 1224 matches (1.4\%). Among the 1224 sources, 412 have the IRAS $12 \mu \mathrm{m}$ flux brighter than $100 \mathrm{mJy}$. This is a sufficiently small number, and we conclude that the extragalactic contaminant has little or insignificant effects on the following analyses.

\section{Results and analyses}

\subsection{Color-color diagrams}

\subsection{1. $(V-S 9 W)$ vs. $(B-V)$}

Figure 4 shows the $(V-S 9 W)$ vs. $(B-V)$ color-color diagram for the AKARI-Hipparcos nearby sources. Only sources with $S / N>5$ in both colors are plotted in the figure. There are 58793 sources that match the criteria.

Be stars and Wolf-Rayet stars. In the color-color diagram, there is a distinct sequence consisting of Wolf-Rayet stars and Be stars, starting at about $(B-V) \sim-0.2$ and $(V-S 9 W) \sim 0$ and extending towards $(B-V) \sim 0.5$ and $(V-S 9 W) \sim 3$, up to $(V-S 9 W) \sim 9$. Some of these types of stars clearly show stronger infrared excess in the $S 9 W$-band than ordinary B- or O-type stars. Thermal emission from dust grains contributes to this infrared excess (Waters et al. 1987; Zhang et al. 2004) in addition to free-free and bound-free radiation from the surrounding nebulosity (e.g., Wright \& Barlow 1975; Chokshi \& Cohen 1987). In our plot, objects with a circumstellar envelope include the Herbig Ae/Be star HD 100546 and the Be star, ८ Ara, which have been identified as Be stars with infrared excess (Waters et al. 1987). There are several objects with similar colors without identifications, suggesting potential candidates of Wolf-Rayet stars and Be stars with infrared excesses.

M-type giants. The M-type giants and carbon stars are clearly separated in the diagram. M-type giants have decreasing $(B-V)$, as $(V-S 9 W)$ becomes redder, while the majority of carbon stars have an increasing trend in both $(B-V)$ and $(V-S 9 W)$, although there is some scatter among carbon stars. This color-color diagram is useful for separating two chemical types of AGB stars.
M-type giants have decreasing $(B-V)$, as $(V-S 9 W)$ becomes redder, because of the anti-correlation of $(B-V)$ and spectral indices of M-type giants. Figure 5 shows the relations between the colors and the spectral subclass indices of M-type giants. The spectral indices may have a range, such as M3/M4III due to time variations of spectral types, and we took the mean values in such cases. It is clear that $(B-V)$ and $(V-S 9 W)$ colors show a different response to the increase in spectral index, such that $(B-V)$ becomes bluer with increasing $M$ spectral index, while $(V-S 9 W)$ becomes gradually redder. By definition, the spectral subclass indices represent the effective temperature $\left(T_{\text {eff }}\right)$ of the star. Based on the model atmosphere, Bessel et al. (1998) showed that the $(B-V)$ color of M-type giants anti-correlates with $T_{\text {eff }}$. This trend is found in stars with solar metallicity or above, while a linear-correlation is found for subsolar metallicity stars. Andrews (1975) suggested that, for M-type giants, the suppression of fluxes due to $\mathrm{TiO}$ molecules is not as strong in $B$-band as in the $V$-band. As $T_{\text {eff }}$ decreases, the $\mathrm{TiO}$ band absorption is expected to become stronger and to suppress the $V$-band flux of M-type giants. It thus seems that the $\mathrm{TiO}$ absorption can explain the trend of M-type giants being bluer in $(B-V)$ colors with increasing spectral indices.

M-type supergiants. There are six M-type supergiants isolated around $7<(V-S 9 W)<9.5$ and $2<(B-V)$, and additional three stars are found in the similar color-region, which can be M-type supergiants. These M-type supergiants have much redder $(B-V)$ colors than those of M-type giants at any given $(V-S 9 W)$ color. What makes M-type supergiants redder in $(B-V)$ color than those of M-type giants? Interstellar extinction may play some role. However, other early-type stars (i.e., O, and B stars, which are located around $(V-S 9 W, B-V) \sim(0,0))$, which can be also affected by their nearby clouds, do not seem to be reddened so much. There should be other reasons. One possible explanation is that the effective surface temperatures of M-type supergiants are generally lower than those of M-type giants, if compared with the same spectral type. For example, Cox (2000) lists $T_{\text {eff }}$ of $3380 \mathrm{~K}$ for the M5 giant, but $2880 \mathrm{~K}$ for the M5 supergiant. However, lower $T_{\text {eff }}$ tends to make the $(B-V)$ color of M-type giants bluer as discussed above, and one could imagine that the situation is almost the same for M-type 


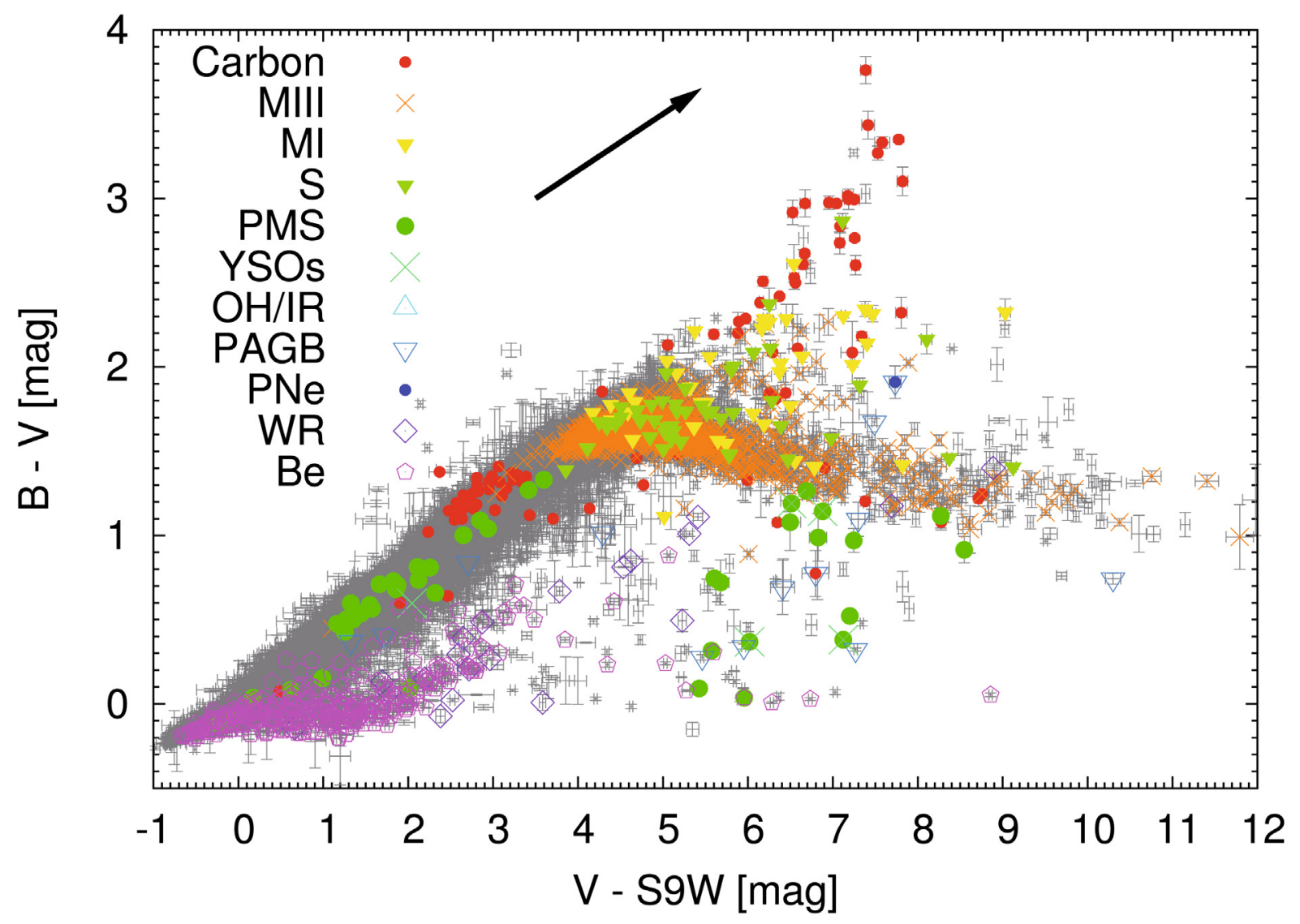

Fig. 4. The $(V-S 9 W)$ vs. $(B-V)$ color-color diagram. The error bars show $\pm 1 \sigma$ errors in colors. Only sources with $S / N>5$ in both colors are plotted. There are 58793 sources that match the criteria. Symbols indicate object types as described in the figure, and gray dots (with error bars) show colors of objects without known identifications. Carbon, MIII, MI, S, PMS, YSOs, OH/IR, PAGB, PNe, WR, and Be represent carbon stars, M-type giants, M-type supergiants, S-type stars, pre-main sequence stars, young stellar objects, $\mathrm{OH} / \mathrm{IR}$ stars, post-AGB stars, planetary nebulae, Wolf-Rayet stars, and Be stars. Occasionally, more than one object type are found for a single object in the SIMBAD database. In that case, we show all of the object types. The black arrow shows the interstellar extinction vector for $A_{v}=2 \mathrm{mag}$, using the Weingartner \& Draine (2001) Milky Way model of $R_{v}=3.1$.

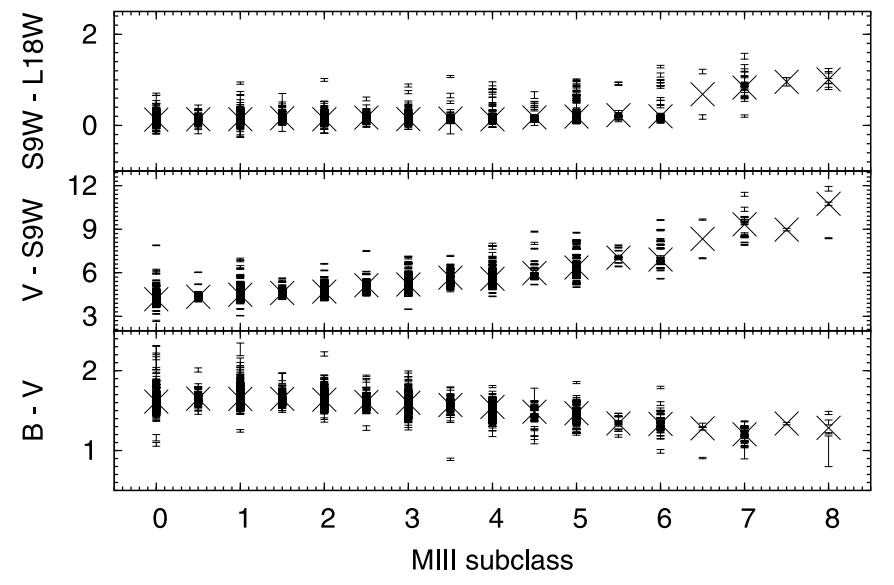

Fig. 5. Correlation between colors and spectral indexes of M-type giants. Only sources with $S / N>5$ in each color are shown. The error bars show $\pm 1 \sigma$ errors in colors. The big crosses show median values for the colors of each spectral index.

supergiants. This contradiction may be understood by the difference in surface gas density between giants and supergiants, because the surface gas density of M-type supergiants is generally lower than that of M-type giants (Cox 2000). Therefore, at a given $T_{\text {eff }}, M$-type supergiants should be less effective in forming the molecules that contribute to absorb $V$-band flux (e.g., TiO). The other possibility comes from line blanketing in the $B$-band. Massey (2002) writes, "at a given $(V-R)$ color, a low-gravity star will have a larger $(B-V)$ value than a higher gravity star, as a result of the increased importance of line blanketing at lower surface gravities, which is most pronounced in the B-band because of the multitude of weak metal lines in the region", and as one might expect, the surface gravity of the M-type supergiant is lower by more than 1 dex than that of the M-type giant (e.g., Cox 2000). The influence of the circumstellar dust is also a possibility. The separation of M-type supergiants from M-type giants can be the result of a combination of these factors. Some M-type supergiants seem to overlap with M-type giants in Fig. 4. We suspect that they are misidentified and are acutually M-type giants, not supergiants (see also Sect. 3.2).

\subsection{2. (S9W - L18W) vs. (J - L18W)}

Figure 6 shows the $(S 9 W-L 18 W)$ vs. $(J-L 18 W)$ color-color diagram using only infrared wavelength bands. We found more cross-identifications between AKARI-2MASS than between AKARI-Hipparcos. In this figure, not only nearby Hipparcos sources, but also more distant sources in the Milky Way, and some extragalactic point sources (e.g., red supergiants in the LMC) are present. However, the vast majority of the sources 
Y. Ita et al.: AKARI's infrared view on nearby stars
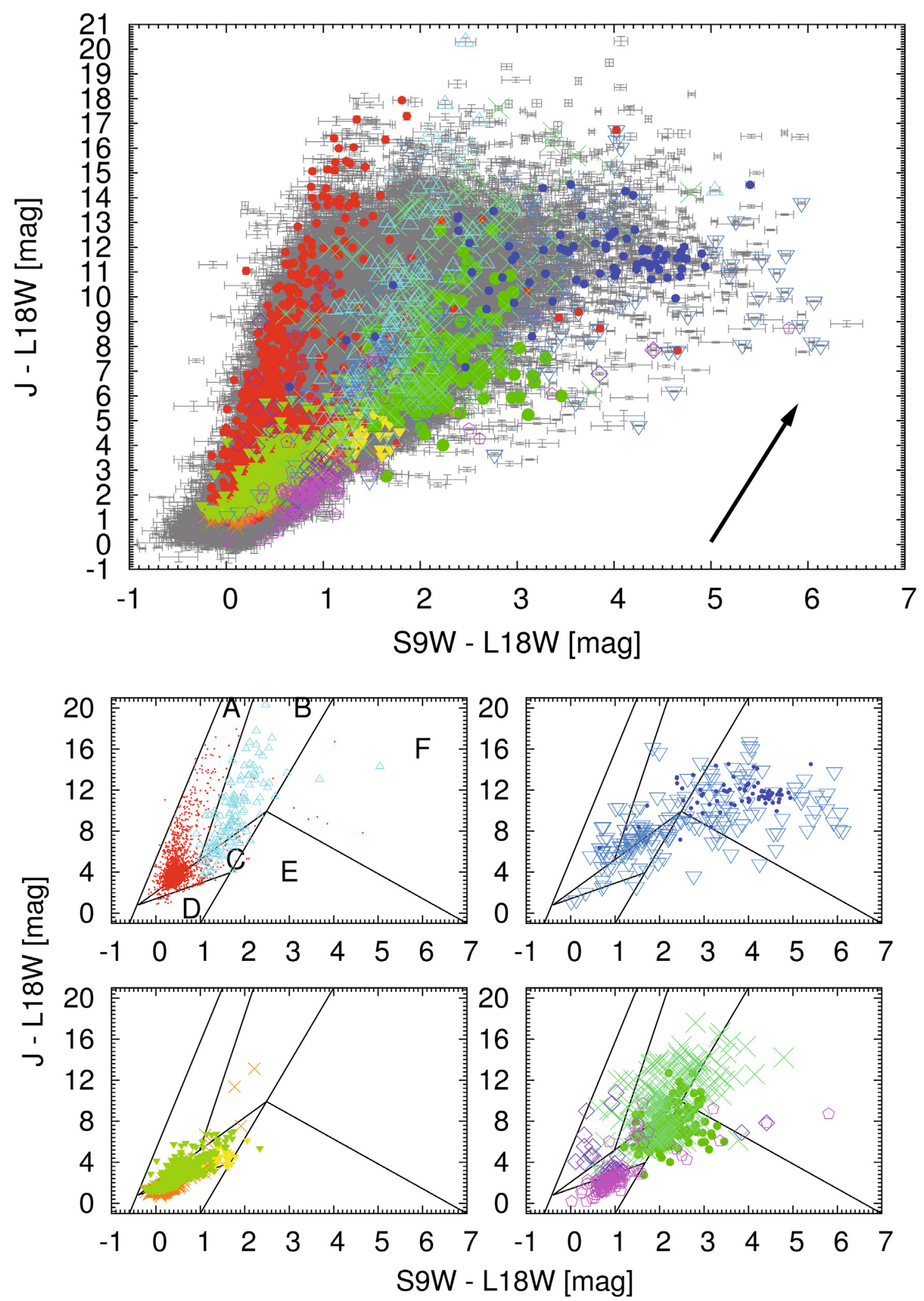

Fig. 6. Upper panel: the $(S 9 W-L 18 W)$ vs. $(J-L 18 W)$ color-color diagram. The error bars show $\pm 1 \sigma$ errors in colors. Only sources with $S / N>5$ in both colors are included. There are 117576 sources that match the criteria. Symbols are the same as in Fig. 4. The black arrow shows the interstellar extinction vector for $A_{v}=20 \mathrm{mag}$, using the Weingartner \& Draine (2001) Milky Way model of $R_{v}=3.1$. Lower panel: the same diagram as in the upper panel, but only including sources with known object types. Black lines show the approximate boundaries of different types of objects: middle-left: carbon stars and OH/IR stars; middle right: PNe and post-AGB stars; bottom-left: M-type giants and supergiants, and S-type stars; bottom-right: YSOs, PMSs, WR stars and Be stars (see text). 


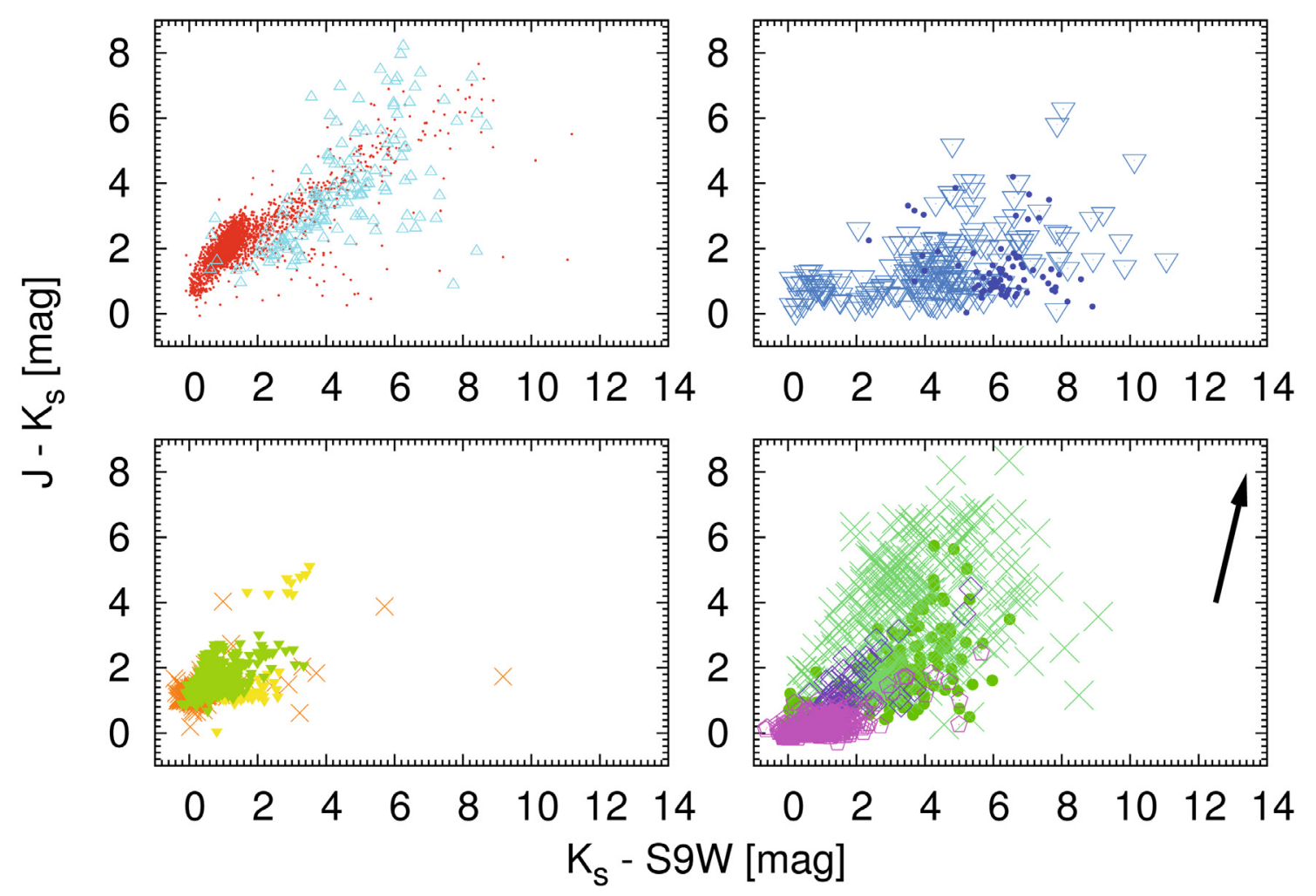

Fig. 7. The $\left(K_{\mathrm{s}}-S 9 W\right)$ vs. $\left(J-K_{\mathrm{s}}\right)$ color-color diagrams of sources with known object types. Only sources with $S / N>5$ in both colors are included. Symbols are the same as in Fig. 4. The black arrow shows the interstellar extinction vector for $A_{v}=20$ mag, using the Weingartner \& Draine (2001) Milky Way model of $R_{v}=3.1$.

should be galactic, and extragalactic ones do not affect any of the discussions below.

Red giants. Carbon stars and oxygen-rich stars (M-type giants, supergiants, and $\mathrm{OH} / \mathrm{IR}$ stars) are separated well in this diagram. This separation is mainly due to the different dust properties between carbon stars and oxygen-rich stars, although molecular bands are the main cause of the separation for relatively blue red giants. Carbon stars show a band attributed to $\mathrm{SiC}$ at $11.3 \mu \mathrm{m}$, in addition to amorphous carbon dust, which has continuous emission without a characteristic feature in the infrared and which dominates dust mass and infrared excess in carbon stars (Groenewegen et al. 1995). Carbon stars with a higher mass-loss rate become red, owing to amorphous carbon dust (Matsuura et al. 2009). The $\mathrm{SiC}$ feature falls in the $S 9 \mathrm{~W}$-band (see Fig. 1), and it is usually found in emission: only a few carbon stars are known to have the $\mathrm{SiC}$ feature in absorption (Speck et al. 1997; Pitman et al. 2006; Gruendl et al. 2008; Speck et al. 2009). Thus carbon stars tend to increase monotonously in both $(S 9 W-L 18 W)$ and $(J-L 18 W)$ colors.

In contrast, heavily mass-losing oxygen-rich stars show the silicate bands at $9.8 \mu \mathrm{m}$ and $18 \mu \mathrm{m}$. In Fig. 6, OH/IR stars contribute to the distinct sequence among all oxygen-rich stars. Usually $\mathrm{OH} / \mathrm{IR}$ stars have the silicate $9.8 \mu \mathrm{m}$ feature in (deep) absorption, while the $18 \mu \mathrm{m}$ silicate band remains in emission (in exceptional cases in absorption; Sylvester et al. 1999). Therefore, $\mathrm{OH} / \mathrm{IR}$ stars are slightly redder in $(S 9 W-L 18 W)$ than carbon stars.

For relatively blue red giants, molecular feature is the reason that locates carbon stars, M-type giants, supergiants, and S-type stars in different regions of the color-color diagram. Carbon stars have $\mathrm{C}_{2}$ and $\mathrm{CN}$ absorption bands in $J$-band (Loidl et al. 2001), suppressing $J$-band fluxes. The wing of broad and strong $\mathrm{C}_{2} \mathrm{H}_{2}$ and $\mathrm{HCN}$ band is found (Matsuura et al. 2007) in the $S 9 W$-band. M-type giants and supergiants show $\mathrm{CO}$ and $\mathrm{TiO}$ absorption bands, and supergiants also have $\mathrm{CN}$ bands in the $J$-band (Lançon et al. 2007), while M-type giants and supergiants have weak molecular features within the $S 9 W$-band, such as $\mathrm{CO}_{2}$ (Justtanont et al. 1998). In S-type stars, the major molecular features are $\mathrm{CO}$, but weak features of carbon-bearing or oxygen-bearing molecules could be found, depending on the $\mathrm{C} / \mathrm{O}$ ratio (Hony et al. 2009). All of these effects cause the characteristic colors of these groups.

Figure 7 is another infrared color-color diagram for sources with known object types similar to the Fig. 6. Only sources with $S / N>5$ in the colors in question are plotted. This figure demonstrates how the separations in the color-color diagram between the object types would be if we emphasize the near-infrared photometry. Now the separation between the carbon stars and the OH/IR stars is blurred, highlighting the importance of the AKARI data for the characterization of red giants. It can be emphasized that this diagram is useful for selecting mass-losing $(J-K>4.0)$ M-type supergiants from M-type giants.

Young stellar objects and pre-main-sequence stars. YSOs and PMS stars are well separated from AGB stars in the color-color diagram, although PNe and post-AGB stars are found to have similar colors. YSOs and PMS stars have lower luminosity in $L 18 \mathrm{~W}$-band than other types of stars with similar infrared excess. YSOs and PMS stars are marginally separable in Fig. 6, in the sense that the latter group has relatively bluer $(J-L 18 W)$ color. Because we could not find any criteria to distinguish YSOs from PMS stars in Wenger et al. (2000), here we assume that YSO are embedded Class I or II objects, and PMS stars represent more evolved Class III objects (Lada \& Wilking 1984). A marginal separation between the two groups is 
Table 5. Number of sources in each region.

\begin{tabular}{lrrrrrrrrrrrr}
\hline \hline Region & \multicolumn{1}{c}{ Known object type } & & & \multicolumn{1}{c}{ Unidentified } \\
& Carbon & MIII & MI & S & PMS & YSOs & OH/IR & PAGB & PNe & WR & Be & \\
\hline A & 1441 & 1 & 0 & 37 & 0 & 11 & 6 & 15 & 1 & 9 & 2 & 9530 \\
B & 27 & 4 & 0 & 5 & 19 & 88 & 90 & 44 & 9 & 0 & 5 & 14162 \\
C & 548 & 75 & 50 & 344 & 58 & 45 & 27 & 20 & 0 & 12 & 11 & 62958 \\
D & 36 & 812 & 12 & 117 & 1 & 0 & 0 & 3 & 0 & 10 & 92 & 23940 \\
E & 4 & 0 & 3 & 1 & 72 & 35 & 2 & 19 & 1 & 0 & 6 & 1089 \\
F & 6 & 0 & 0 & 0 & 6 & 19 & 2 & 61 & 65 & 2 & 3 & 1336 \\
\hline
\end{tabular}

probably because the circumstellar disks become optically thin, and the central stars become visible in PMS stars.

Unidentified objects. In the lower panel of Fig. 6, we defined six regions to make an approximate object classification on the color-color diagram. The representing object types in the regions are (A) Red carbon stars; (B) OH/IR stars and some YSOs; (C) M-type giants, supergiants and S-type stars, as well as bluer carbon stars in the blue part, and PMS stars in the red part; (D) Be stars, M-type giants, and S-type stars; (E) PMS stars, PNe, and PAGB stars; (F) PNe and PAGB stars. Objects in regions $\mathrm{A}$ and $\mathrm{B}$ are most likely to be carbon stars and $\mathrm{OH} / \mathrm{IR}$ stars, respectively, while $\mathrm{PNe}$, post-AGB stars, and YSOs tend to spread over the diagram, and it is difficult to identify these types of stars only with these infrared colors. Region D is mixed up with Be stars and red giants (M-type giants/supergiants and S-type stars). However, these two populations can be easily separated by their optical color, such as $(B-V)$ (see Fig. 4).

A comparison of the two panels of Fig. 6 indicates that there are many infrared stars without object type classifications. Some objects fall into regions $\mathrm{A}$ and $\mathrm{B}$, and they are likely to be extremely red carbon stars and $\mathrm{OH} / \mathrm{IR}$ stars, respectively. To explore these unidentified objects, we are now conducting followup observations, using the AKARI during post-helium mission, which take $2.5-5 \mu \mathrm{m}$ spectra with $\lambda / \Delta \lambda \sim 100$ for selected sources redder than $f_{S 9 W} / f_{K_{\mathrm{s}}}>2$ in $|b|>30^{\circ}$ (PI: S. Oyabu) and also for selected sources in $|b|<30^{\circ}$ (PI: D. Ishihara), where $f_{S 9 W}$ is $S 9 \mathrm{~W}$ flux in Jy, $f_{K_{\mathrm{s}}}$ is 2 MASS's $K_{\mathrm{s}}$-band flux in Jy, and $b$ is the galactic latitude, respectively. In Table 5, we summarize the number of sources with known object type, and unidentified sources in each region.

\subsection{Color-magnitude diagram}

Nearby objects in the Galaxy have the advantage of allowing us to make a detailed study of them, while we may have difficulty in correctly estimating the distance and thus in obtaining the absolute magnitude. Objects in nearby galaxies, on the other hand, have similar distances, so it is fairly straightforward to make a color-magnitude diagram (CMD) for them. The Hipparcos data have changed the situation drastically and allow us to estimate the distance of nearby objects reliably. By combining the AKARI All-Sky Survey data with the Hipparcos data, we are able to make a mid-infrared CMD for Galactic objects whose nature is understood. Comparing the AKARI CMD with those of the LMC obtained by the Spitzer SAGE program (Meixner et al. 2006) enables us to investigate populations in the LMC CMD, for which little information is available, such as "fainter, redder O-rich giants" (Blum et al. 2006; Srinivasan et al. 2009).

\subsubsection{General overview}

Figure 8 shows the $(S 9 W-L 18 W)$ vs. $M_{L 18 W}$ infrared CMD, where $M_{L 18 W}$ is the absolute magnitude in $L 18 W$-band. Only sources with $\sigma \omega / \omega<0.4$ and $S / N>5$ in $(S 9 W-L 18 W)$ color are included in the figure, where $\omega$ and $\sigma \omega$ are the parallax and its error, respectively. There are 13252 sources that matches these criteria. In our data set, the brightest stars in $M_{L 18 W}$ are post-AGB stars, followed by M-type supergiants and giants, carbon stars, and S-type stars. The YSO and PMS stars show large excess in the color $1.5<(S 9 W-L 18 W)<3$. The Be stars tend to have lower $M_{L 18 W}$ luminosities with moderate $0.3<(S 9 W-L 18 W)<1.3$ excess, and WR stars also have similar magnitudes and colors as Be stars. M-type giants follow a sequence of $(S 9 W-L 18 W) \sim 0.1$ from $M_{L 18 W}=-3$ to -8 , and these stars have little emission from the circumstellar envelopes. Once circumstellar envelopes are developed, M-type giants become redder in color. There may be two sequences in M-type giants. One sequence follows $M_{L 18 W}=-9$ mag up to $(S 9 W-L 18 W) \sim 1.5$ and the other follows $M_{L 18 W}=-7 \mathrm{mag}$ up to $(S 9 W-L 18 W) \sim 1.2$. It is not clear whether these two sequences actually represent different populations or stars with different dust properties, or a continuous sequence with a large scatter due to the complexity of dust and molecular features. Carbon stars tend to follow a similar trend to M-type giants with little excess. We have to interpret this CMD cautiously, as there is no parallax available for stars with heavily obscured central stars with circumstellar dust (i.e., heavily mass-losing infrared AGB stars).

\subsubsection{M-type supergiants}

There are six faint $\left(M_{L 18 W}>-7 \mathrm{mag}\right)$ sources classified as M-type supergiants in Fig. 8: KT Mus, HD 306799, RV Pup, V408 Aur, NSV 25773, and KN Cas. Their $M_{L 18 W},(V-S 9 W)$ and $(B-V)$ values are tabulated in Table 7 . Even if they do not have circumstellar dust, they are still too faint to classify as supergiants. They are more likely to be M-type giants, judging from their $L 18 W$ luminosities. Furthermore, all of them have $(V-S 9 W)$ and $(B-V)$ colors similar to the general colors of M-type giants (see Sect. 3.1.1).

\subsubsection{Young stellar objects and pre-main-sequence stars}

The YSO and PMS candidates can be selected in Fig. 8. In that figure, these types of stars are found at $(S 9 W-L 18 W)>1$, although some contamination of other populations, such as post-AGB stars and PNe, is expected. There are 16 YSOs or PMS stars in Fig. 8. The number is mostly limited by the Hipparcos detection limit and parallax errors, and stars without apparent central stars in the optical are not found in this diagram. 


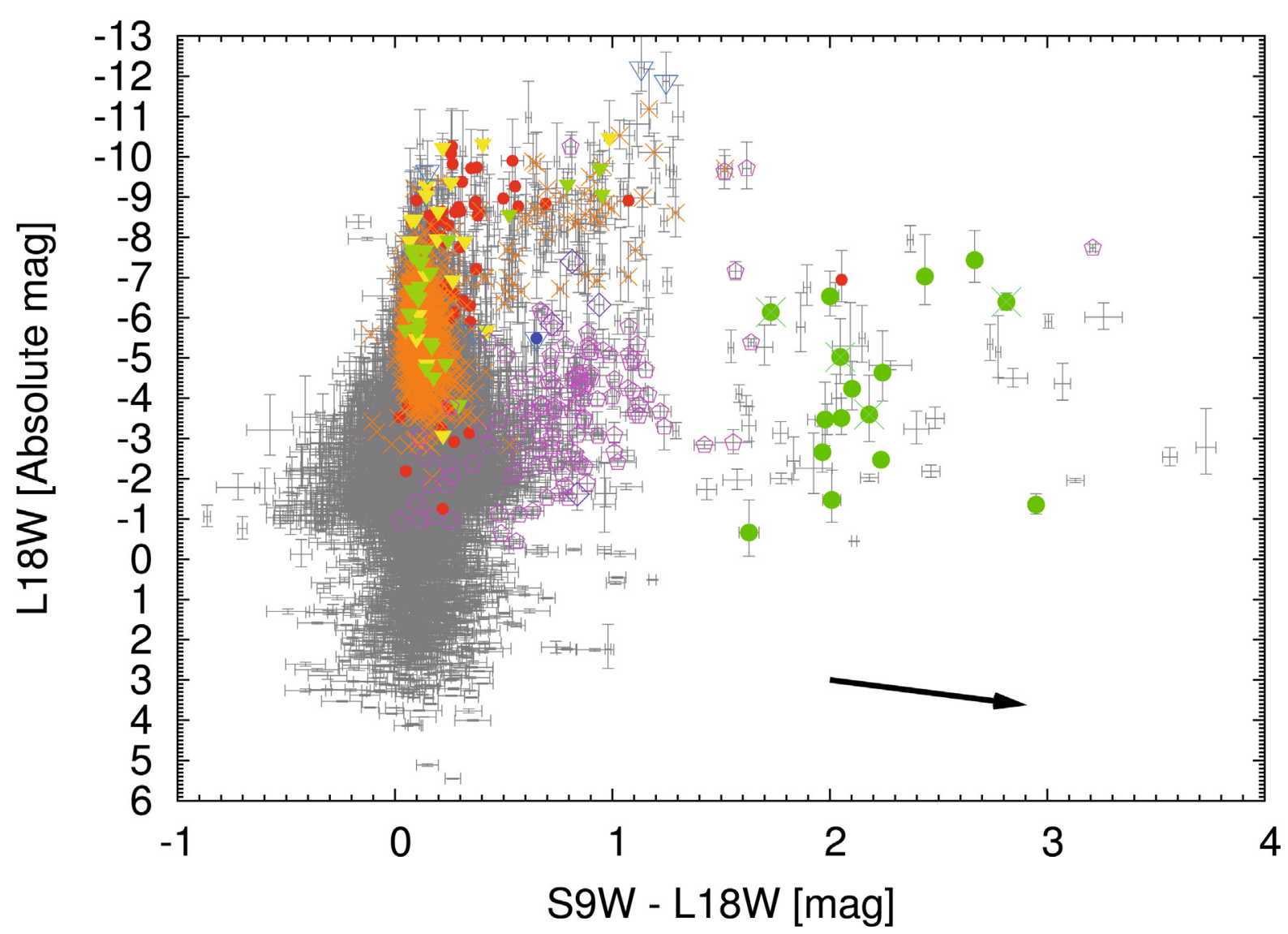

Fig. 8. The $(S 9 W-L 18 W)$ vs. $M_{L 18 W}$ color-magnitude diagram. The error bars show $\pm 1 \sigma$ errors. The vertical error bars include errors in parallax. Only sources with $S / N>5$ in color, and with $\sigma \omega / \omega<0.4$ are shown, where $\omega$ is the Hippparcos parallax. There are 13252 sources that match the criteria. Symbols are the same as in Fig. 4. The black arrow shows the interstellar extinction vector for $A_{v}=20$ mag, using the Weingartner \& Draine (2001) Milky Way model of $R_{v}=3.1$.

Their common names, celestial coordinates, $(S 9 W-L 18 W)$ colors, and $M_{L 18 W}$ absolute magnitudes are listed in Table 6.

A bibliographical survey shows that these stars are $\mathrm{T}$ Tauri and Herbig Ae/Be stars. It is clear that all of them show infrared excess, and the excess should originate in the dust emission in their circumstellar disk (e.g., Whitney et al. 2003; Adam et al. 1987). These stars are distributed in a relatively narrow range in $(S 9 W-L 18 W)$ color (about $1 \mathrm{mag}$ ), while a wider range in $M_{L 18 W}$ luminosity (about $7 \mathrm{mag}$ ). Most of these stars are likely to have disks. The cause of the spread in luminosities $\left(M_{L 18 W}\right)$ is not clear from the table; however, we suggest possibilities, such as the differences in the viewing angle of the disk (Adam et al. 1987), the inner radius of the disk, and the disk mass. It appears that luminosities $\left(M_{L 18 W}\right)$ do not correlate with the stellar mass in our sample.

\subsubsection{Comparison with the Spitzer's color-magnitude diagram in the LMC}

AKARI's mid-infrared CMD helps for understanding ([8.0]-[24]) vs. $M_{24}$ color-magnitude diagram of Spitzer Space Telescope photometric data, such as those from Magellanic Clouds catalog (LMC: Meixner et al. 2006; SMC: Gordon et al. 2010, in prep.).

We compared our $(S 9 W-L 18 W)$ vs. $M_{L 18 W}$ diagram of galactic objects with the Spitzer ([8.0]-[24]) vs. $M_{24}$ diagram of the LMC sources as shown in Fig. 9. After considering the offset values given in the Appendix, $(S 9 W-L 18 W) \sim 1$ should correspond to ([8.0]-[24]) 1.7. Therefore the galactic M-type giants, carbon stars, and S-type stars with infrared excess $(0.4<$ ( $S 9 W-L 18 W)<1.5$ and $M_{L 18 W}<-6$ in absolute magnitude) probably correspond to the LMC fainter, redder sources located below the solid line (i.e., sources located on or below the sequence "D") indicated in Fig. 93, which is marked in Blum et al. (2006) and Srinivasan et al. (2009).

We looked into the properties of the galactic less luminous (although they are among the brightest sample in our data set) red giants as the counterparts to the fainter, redder sources in the LMC. We extracted M-type giants, carbon stars, and S-type stars that satisfy $(S 9 W-L 18 W)>0.4$ and $M_{L 18 W}<-6$. There are 4 S-type stars, 7 carbon stars, and 38 M-type giants that match the criteria. Then, we checked their pulsation properties (variability type and pulsation period), and also searched for their ISO/SWS spectra. The results are summarized in Table 8. We find that all but one (HIP 56551) stars are known variable stars. Most of them show irregular or semi-regular type light variations. Judging from their relatively long pulsation periods, it is likely that they are on the AGB, because faint variables with luminosities at, around, or below the tip of the first red giant branch (RGB) have shorter periods of about 30 days (e.g., Ita et al. 2004). Among the 49 samples listed in Table 8, the ISO/SWS spectra (Sloan et al. 2003a) are available for 7 stars. These spectra are shown in Fig. 10 with their names and

\footnotetext{
3 The names of the sequences in Fig. 9 are irrelevant to the region names defined in Fig. 6.
} 
Table 6. Young stellar objects or pre-main sequence stars plotted in Fig. 8.

\begin{tabular}{|c|c|c|c|c|c|c|c|c|c|c|}
\hline $\begin{array}{l}\text { Common } \\
\text { name }\end{array}$ & $\begin{array}{r}\mathrm{RA} \\
\text { degree }\end{array}$ & $\begin{array}{r}\text { Dec } \\
(\mathrm{J} 2000)\end{array}$ & $\begin{array}{r}S 9 W-L 18 W \\
\text { (mag) }\end{array}$ & $\begin{array}{r}M_{L 18 W} \\
\text { (abs. mag) }\end{array}$ & Type & $\begin{array}{l}\log T_{\text {eff }} \\
(\mathrm{K})\end{array}$ & $\begin{array}{l}L_{\star} \\
\left(L_{\odot}\right)\end{array}$ & $\begin{array}{l}\text { Mass } \\
\left(M_{\odot}\right)\end{array}$ & $\begin{array}{l}\text { log (Age) } \\
\text { (yrs) }\end{array}$ & Ref. \\
\hline V773 Tau & 63.55383 & 28.20341 & 1.97 & -2.66 & WT & 3.695 & 7.61 & 1.74 & 5.71 & $\mathrm{~b}, \mathrm{c}$ \\
\hline $\mathrm{T} \mathrm{Tau}$ & 65.49764 & 19.53512 & 2.67 & -7.43 & TT & 3.708 & 14 & 1.91 & 5.77 & $\mathrm{~b}, \mathrm{c}$ \\
\hline DF Tau & 66.76165 & 25.70620 & 1.63 & -0.66 & TT & 3.587 & 2.97 & 0.53 & 5.00 & $\mathrm{~b}$ \\
\hline GW Ori & 82.28496 & 11.87019 & 2.44 & -7.03 & TT & & & & & $\mathrm{b}$ \\
\hline HD 36112 & 82.61471 & 25.33252 & 2.00 & -6.53 & AB cand. & 3.91 & 1.35 & 2.0 & 6.5 & $\mathrm{a} 2$ \\
\hline CR Cha & 164.77906 & -77.02787 & 2.18 & -3.59 & TT & & & & & $\mathrm{b}$ \\
\hline TW Hya & 165.46628 & -34.70473 & 2.95 & -1.36 & TT & & & & & $\mathrm{b}$ \\
\hline DI Cha & 166.83634 & -77.63536 & 2.05 & -5.03 & TT & & & & & $\mathrm{b}$ \\
\hline CU Cha & 167.01383 & -77.65489 & 2.81 & -6.39 & $\mathrm{AB}$ cand. & 4.00 & 1.61 & 2.5 & $>6.3$ & $a 2, b$ \\
\hline HD 97300 & 167.45841 & -76.61326 & 1.73 & -6.14 & $\mathrm{AB}$ & 4.02 & 1.54 & 2.5 & $>3$ & a1 \\
\hline HT Lup & 236.30362 & -34.29184 & 2.10 & -4.23 & WT & 3.699 & 4.69 & 2 & $6-6.5$ & $\mathrm{e}$ \\
\hline HD 141569 & 237.49062 & -3.92121 & 2.24 & -2.47 & AB cand. & 4.00 & 1.35 & 2.3 & $>7.0$ & $\mathrm{a} 2$ \\
\hline RY Lup & 239.86828 & -40.36423 & 1.98 & -3.47 & $\mathrm{TT}$ & & & & & $\mathrm{b}$ \\
\hline V1121 Oph & 252.31377 & -14.36906 & 2.24 & -4.63 & TT & & & & & $\mathrm{b}$ \\
\hline AK Sco & 253.68687 & -36.88849 & 2.05 & -3.51 & AB cand./TT & 3.81 & 0.88 & & & $\mathrm{a} 2, \mathrm{~b}$ \\
\hline FK Ser & 275.09479 & -10.18712 & 2.01 & -1.47 & TT & & & & & $\mathrm{b}$ \\
\hline
\end{tabular}

Notes. $L_{\star}$ : luminosity of the central star; types: WT: Weak-lined T Tauri; TT: T Tauri; AB: Herbig Ae/Be; cand.:candidates.

References. a1: van den Ancker et al. (1997); a2: van den Ancker et al. (1998); b: Bertout et al. (1999); c: Beckwith et al. (1990); e: Gras-Velazquez \& Ray (2005).

Table 7. These stars are classified as M-type supergiants in the SIMBAD, but probably M-type giants.

\begin{tabular}{lrrr}
\hline \hline Name & $M_{L 18 W}$ & $\begin{array}{c}(V-S 9 W) \\
{[\mathrm{mag}]}\end{array}$ & $(B-V)$ \\
\hline KT Mus & -6.94 & 6.50 & 1.77 \\
HD 306799 & -4.85 & 4.60 & 1.85 \\
RV Pup & -5.70 & 6.79 & 1.41 \\
V408 Aur & -6.08 & 5.45 & 1.79 \\
NSV 25773 & -5.49 & 4.40 & 1.69 \\
KN Cas & -3.08 & 5.65 & 1.57 \\
\hline
\end{tabular}

classification indices defined in Kraemer et al. (2002). According to their classification, group 2 includes sources with SEDs dominated by the stellar photosphere but also influenced by dust emission. The SE and CE subgroups correspond to the oxgen-rich dust emission and carbon-rich dust emission, respectively. The M subgroup denotes "miscellaneous". It is clear that all of the stars are surrounded by optically-thin circumstellar dust shells. Silicate dust features are seen in almost all M-type giants and S-type stars. Also, $\mathrm{SiC}$ feature at around $11.3 \mu \mathrm{m}$ can be seen in carbon stars. Interestingly, the so-called " $13 \mu \mathrm{m}$ feature" is seen in M-type and/or S-type giants. This feature is probably due to aluminum oxides (e.g., Posch et al. 1999). Sloan et al. (2003b) suggests that this feature tends to be stronger in systems with lower infrared excesses and thus lower mass-loss rates (e.g., Onaka et al. 1989; Kozasa \& Sogawa 1997). Interestingly, Sloan et al. (1996) reported that the $13 \mu \mathrm{m}$ feature is preferentially detected in semi-regular or irregular variables. Based on these available data of galactic counterparts, we suggest that low mass-loss rate M-type giants, S-type stars, and carbon stars are mixed up together to make sequence "D" in the LMC (Fig. 9).

The counterparts to the sources on the brighter part ([24] < -13) of sequence "B" are not present in AKARI $(S 9 W-L 18 W)$ vs. $M_{L 18 W}$ color-magnitude diagram, owing to the lack of reliable parallax measurements, and/or such bright sources are saturated either in $S 9 W$ or $L 18 \mathrm{~W}$ measurements.

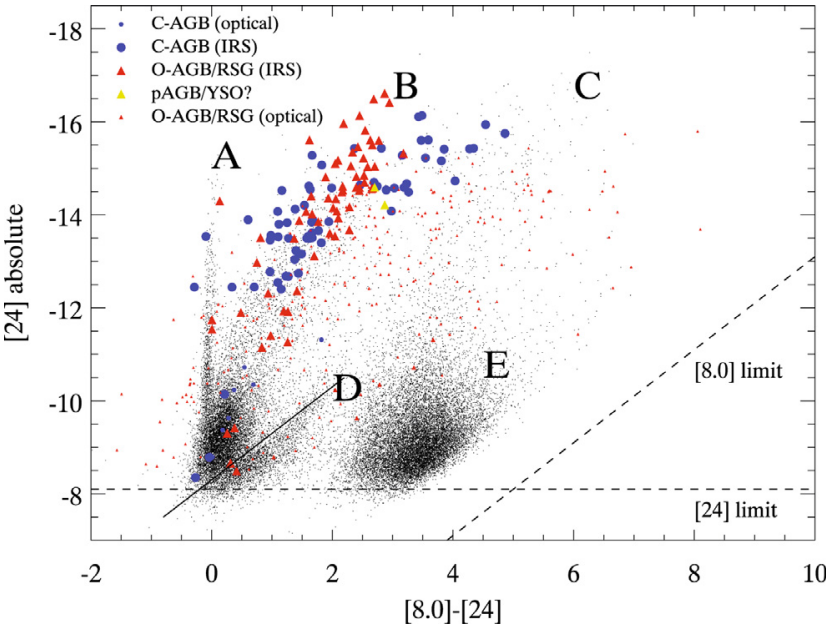

Fig. 9. The ([8.0]-[24]) vs. $M_{24}$ color-magnitude diagram of sources in the LMC, with a distance modulus of $18.5 \mathrm{mag}$ adopted for the LMC sources. Symbols show oxygen-rich or carbon-rich AGB stars and red supergiants whose chemical types are spectroscopically confirmed, taken from Matsuura et al. (2009). Sequence "A" is the foreground stars (not belong to the LMC). Sequence "B" contains both oxgen-rich and carbon-rich AGB stars, and also contains heavily mass-losing stars at the tip ([24] $<-15 \mathrm{mag}$ ). The solid line is defined by Srinivasan et al. (2009), and the sources below the line (sequence marked as "D") are the fainter, redder O-rich population mentioned in Blum et al. (2006), which correspond to the red sequence in $(S 9 W-L 18 W) \sim 1$ and $M_{L 18 W} \sim-9$. It appears both oxygen-rich and carbon-rich stars are found in sequence "D" and "B". Dashed lines show the detection limits of [8.0] and [24] quoted in Meixner et al. (2006).

\section{Summary}

AKARI's mid-infrared all-sky survey has increased the number of known mid-infrared sources drastically, mainly because of better spatial resolution than in previous mid-infrared surveys. We combined the first-release version $(\beta-1)$ of the AKARI IRC 


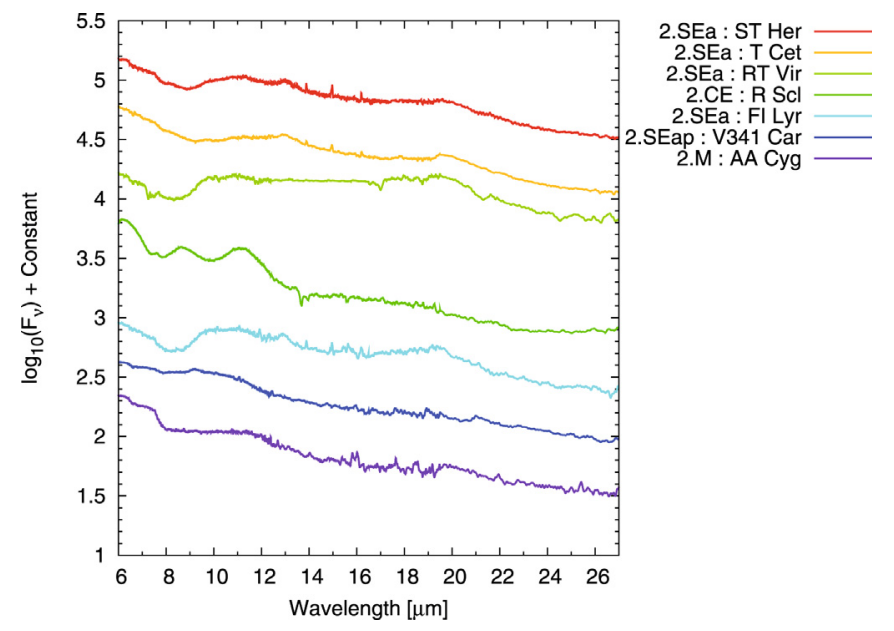

Fig. 10. The ISO/SWS spectra for all sources selected in Sect. 4.2.4. The scale of the vertical axis is arbitrary. Stars are sorted in increasing order of $L 18 \mathrm{~W}$ luminosity from bottom to the top.

All-Sky survey point source catalog with the existing all-sky survey catalogs, namely the Hipparcos and the 2MASS. Two-color diagrams were made with the aim of classifying sources. We found that oxygen-rich giants and carbon stars are separated well by adding AKARI's new data. Also, we showed that Be stars and Wolf-Rayet stars with strong infrared excesses can be effectively selected by using optical and AKARI's combined colors. In conjunction with Hipparcos parallax measurements, we plotted an infrared color-magnitude diagram. We uncovered the properties of redder, fainter red giants in the LMC by comparing their galactic counterparts. This work will be greatly expanded in the forthcoming GAIA era, when we have good parallax measurements for tens of millions of stars. AKARI's new All-Sky Survey data reveal not only the mid-infrared characteristics of known objects, but also the existence of many as yet unidentified infrared sources. The color-color and color-magnitude diagrams we presented can be used to extrapolate the properties of the unidentified objects, leading to a follow-up campaign.

Acknowledgements. We thank the anonymous referee for the constructive comments that helped to improve this paper. This work is based on observations with AKARI, a JAXA project with the participation of ESA. This work is supported by the Grant-in-Aid for Encouragement of Young Scientists (B) No. 21740142 from the Ministry of Education, Culture, Sports, Science and Technology of Japan.This research has made use of the SIMBAD database, operated at the CDS, Strasbourg, France. This publication makes use of data products from the Two Micron All Sky Survey, which is a joint project of the University of Massachusetts and the Infrared Processing and Analysis Center/California Institute of Technology, funded by the National Aeronautics and Space Administration and the National Science Foundation. This research has made use of the NASA/IPAC Extragalactic Database (NED), which is operated by the Jet Propulsion Laboratory, California Institute of Technology, under contract with the National Aeronautics and Space Administration.

\section{Appendix A:}

We demonstrate the conversion between the AKARI $(S 9 W-L 18 W)$ vs. $M_{L 18 W}$ and the Spitzer ([8.0]-[24]) vs. $M_{24}$ color-magnitude diagrams. Using the ISO/SWS fluxcalibrated spectral library (Sloan et al. 2003a), we calculated synthetic magnitudes of AKARI's $S 9 W$ - and $L 18 W$-bands and Spitzer's IRAC [8.0] (Fazio et al. 2004) and MIPS [24]
Table 8. Galactic red giants that are possible counterparts to the fainter, redder giants in the LMC.

\begin{tabular}{|c|c|c|c|c|}
\hline Name & Var. type & Period [days] & $M_{L 18 W}$ & ISO/SWS \\
\hline \multicolumn{5}{|c|}{ Carbon star } \\
\hline $\mathrm{R} \mathrm{Scl}$ & SRB & 370 & -8.96 & $\mathrm{Y}$ \\
\hline TW Hor & SRB & 158 & -8.77 & $\mathrm{~N}$ \\
\hline Y Tau & SRB & 241.5 & -9.27 & $\mathrm{~N}$ \\
\hline V496 Car & SRB & - & -8.84 & $\mathrm{~N}$ \\
\hline HIP 56551 & - & - & -6.94 & $\mathrm{~N}$ \\
\hline${ }^{1}$ FI Lyr & SRB & 146 & -8.91 & $\mathrm{Y}$ \\
\hline U Cyg & Mira & 463.24 & -9.90 & $\mathrm{~N}$ \\
\hline \multicolumn{5}{|l|}{ S-type star } \\
\hline $\mathrm{T}$ Cet & SRC & 158.9 & -9.32 & $\mathrm{Y}$ \\
\hline Y Lyn & SRC & 110 & -9.07 & $\mathrm{~N}$ \\
\hline ST Her & SRB & 148 & -9.71 & $\mathrm{Y}$ \\
\hline AA Cyg & SRB & 212.7 & -8.57 & $\mathrm{Y}$ \\
\hline \multicolumn{5}{|c|}{ M-type giants } \\
\hline $\mathrm{AC} \mathrm{Cet}$ & LB & - & -7.04 & $\mathrm{~N}$ \\
\hline V370 And & SRB & 228 & -9.70 & $\mathrm{~N}$ \\
\hline Z Eri & SRB & 80 & -7.69 & $\mathrm{~N}$ \\
\hline RR Eri & SRB & 97 & -8.38 & $\mathrm{~N}$ \\
\hline VX Eri & SR: & - & -7.02 & $\mathrm{~N}$ \\
\hline SS Cep & SRB & 90 & -8.72 & $\mathrm{~N}$ \\
\hline WX Hor & SRA & - & -8.51 & $\mathrm{~N}$ \\
\hline WW Pic & SRA & - & -6.67 & $\mathrm{~N}$ \\
\hline SW Col & LB: & - & -6.92 & $\mathrm{~N}$ \\
\hline ZZ Pic & LB & - & -6.97 & $\mathrm{~N}$ \\
\hline BQ Ori & SR & - & -6.98 & $\mathrm{~N}$ \\
\hline V341 Car & $\mathrm{L}$ & - & -8.61 & $\mathrm{Y}$ \\
\hline AK Pyx & LB & - & -7.01 & $\mathrm{~N}$ \\
\hline RS Cam & SRB & 88.6 & -6.72 & $\mathrm{~N}$ \\
\hline RT Cnc & SRB & 60 & -8.06 & $\mathrm{~N}$ \\
\hline V489 Car & SRB & - & -8.34 & $\mathrm{~N}$ \\
\hline V496 Car & SRB & - & -8.83 & $\mathrm{~N}$ \\
\hline BB Ant & SRB & 125 & -8.38 & $\mathrm{~N}$ \\
\hline T Crt & SRB & - & -6.65 & $\mathrm{~N}$ \\
\hline ST UMa & SRB & 110 & -9.82 & $\mathrm{~N}$ \\
\hline AZ UMa & LB & - & -8.60 & $\mathrm{~N}$ \\
\hline Z UMa & SRB & 195.5 & -8.52 & $\mathrm{~N}$ \\
\hline RW Vir & LB & - & -8.72 & $\mathrm{~N}$ \\
\hline RT Vir & SRB & 155 & -8.98 & $\mathrm{Y}$ \\
\hline V744 Cen & SRB & - & -8.38 & $\mathrm{~N}$ \\
\hline Y Cen & SRB: & 180 & -9.21 & $\mathrm{~N}$ \\
\hline FY Lib & SRB & 120 & -9.50 & $\mathrm{~N}$ \\
\hline R Ser & Mira & 356.41 & -8.42 & $\mathrm{~N}$ \\
\hline V1293 Aql & SRB & - & -9.02 & $\mathrm{~N}$ \\
\hline V346 Tel & LB & - & -6.36 & $\mathrm{~N}$ \\
\hline V1070 Cyg & SRB & 73.5 & -7.55 & $\mathrm{~N}$ \\
\hline W Cyg & SRB & 131.1 & -9.08 & $\mathrm{~N}$ \\
\hline TW Peg & SRB & 929.3 & -9.77 & $\mathrm{~N}$ \\
\hline BW Oct & LB: & - & -9.02 & $\mathrm{~N}$ \\
\hline DM Tuc & LB & - & -8.56 & $\mathrm{~N}$ \\
\hline Y Scl & SRB & - & -7.68 & $\mathrm{~N}$ \\
\hline CC Ind & SRB: & - & -9.88 & $\mathrm{~N}$ \\
\hline S Phe & SRB & 141 & -8.84 & $\mathrm{~N}$ \\
\hline
\end{tabular}

Notes. (1) This star can be a M-type star (see its ISO spectrum in Fig. 10).

(Rieke et al. 2004). This is the same method as described in Ita et al. (2008). Figure A.1 shows the correlations between calculated $S 9 W$ vs. [8.0], $L 18 W$ vs. [24] and $(S 9 W-L 18 W)$ vs. ([8.0]-[24]). The relation between $S 9 W$ and [8.0] is almost linear, with a median offset $(\equiv[8.0]-S 9 W)$ value of 0.13 mag $(\sigma=0.35 \mathrm{mag})$. There is also a linear correlation between $L 18 \mathrm{~W}$ 

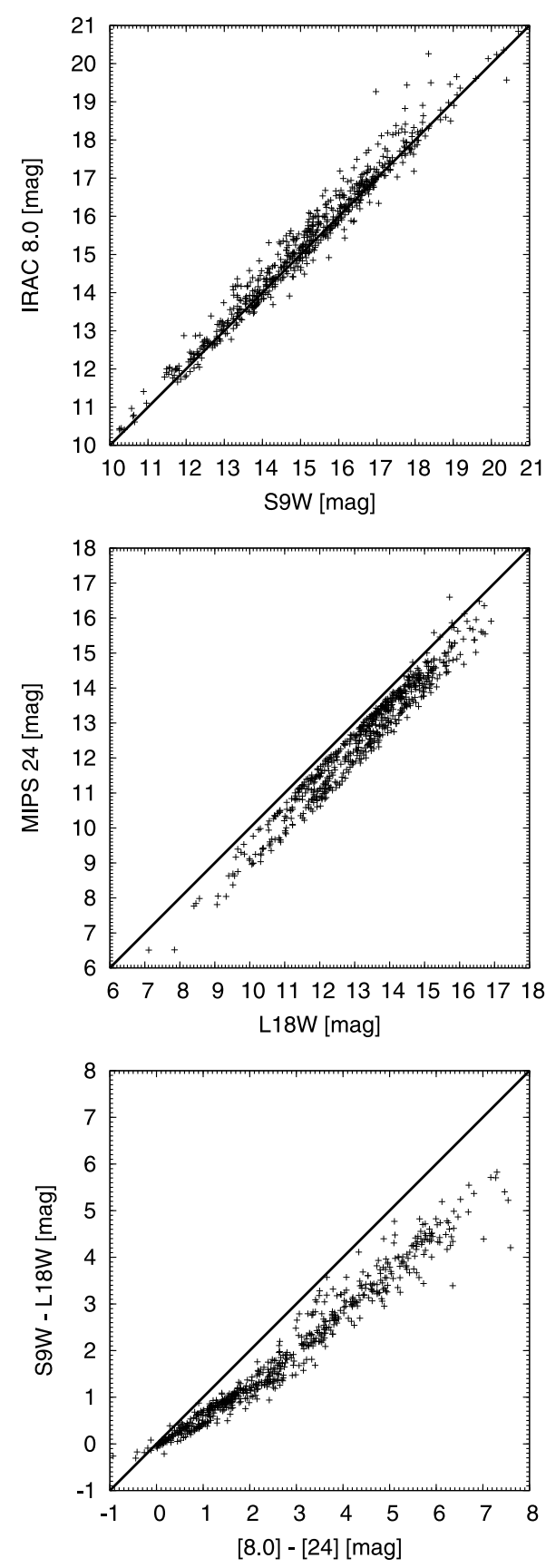

Fig. A.1. The comparison of calculated magnitudes through synthetic photometry on ISO/SWS spectra for $S 9 W$ and IRAC 8.0 (upper panel), L18W and MIPS 24.0 (middle panel), and colors (bottom panel).

and [24] with a notable offset $(\equiv[24]-L 18 W)$ of $-0.62 \mathrm{mag}$ $(\sigma=0.35 \mathrm{mag})$. Actual transformations need color corrections as found in the bottom panel of the figure, but a general trend can be discussed even if we ignore the color correction terms.

\section{References}

Acker, A., Ochsenbein, F., Stenholm, B., et al. 1994, VizieR Online Data Catalog, 5084, 0

Adams, F. C., Lada, C. J., \& Shu, F. H. 1987, ApJ, 312, 788

Andrews, P. J. 1975, MNRAS, 173, 701

Beckwith, S. V. W., Sargent, A. I., Chini, R. S., \& Guesten, R. 1990, AJ, 99, 924

Bessell, M. S., Castelli, F., \& Plez, B. 1998, A\&A, 333, 231

Bertout, C., Robichon, N., \& Arenou, F. 1999, A\&A, 352, 574
Biazzo, K., Frasca, A., Catalano, S., et al. 2006, A\&A, 446, 1129

Blum, R. D., Mould, J. R., Olsen, K. A., et al. 2006, AJ, 132, 2034

Busa, I., Aznar Cuadrad, R., Terranegra, L., et al. 2007, A\&A, 466, 1089

Cenarro, A. J., Peletier, R. F., Sanchez-Blazquez, P., et al. 2007, MNRAS, 374, 664

Chokshi, A., \& Cohen, M. 1987, AJ, 94, 123

Cox, A. 2000, Allen's Astrophysical Quantities, Fourth edition (New-York: Springer-Verlag)

Gras-Velazquez, A., \& Ray, T. P. 2005, A\&A, 443, 541

De Medeiros, J. R., \& Mayor, M. 1999, A\&AS, 139, 433

De Medeiros, J. R., Da Silva, J. R. P., \& Maia, M. R. G. 2002, ApJ, 578, 943

ESA 1997, The Hipparcos catalogue, ESA SP-1200

Fazio, G. G., Hora, J. L., Allen, L. E., et al. 2004, ApJS, 154, 10

Fujiwara, H., Ishihara, D., Kataza, H., et al. 2010, A\&A, submitted

Groenewegen, M. A. T. 1995, A\&A, 293, 463

Gruendl, R. A., Chu, Y.-H., Seale, J. P., et al. 2008, ApJ, 688, L9

Hauser, M. G., Arendt, R. G., Kelsall, T., et al. 1998, ApJ, 508, 25

Hony, S., Heras, A. M., Molster, F. J., \& Smolders, K. 2009, A\&A, 501, 609

IRAS Explanatory Supplement to the Catalogs and Atlases 1988, ed. C. A.

Beichman, G. Neugebauer, \& H. J. Habing, et al. (Washington, DC: GPO)

Ishihara, D., Onaka, T., Kataza, H., et al. 2010, A\&A, 514, A1

Ita, Y., Tanabé, T., Matsunaga, N., et al. 2004, MNRAS, 347, 720

Ita, Y., Onaka, T., Kato, D., et al. 2008, PASJ, 60, S435

Justtanont, K., Feuchtgruber, H., de Jong, T., et al. 1998, A\&A, 330, L17

Kawada, M., Baba, H., Barthel, P. D., et al. 2007, PASJ, 59, S389

Kozasa, T., \& Sogawa, H. 1997, Ap\&SS, 251, 165

Kraemer, K. E., Sloan, G. C., Price, S. D., et al. 2002, ApJS, 140, 389

Lada, C. J., \& Wilking, B. A. 1984, ApJ, 287, 610

Lançon, A., Hauschildt, P. H., Ladjal, D., \& Mouhcine, M. 2007, A\&A, 468, 205 Li, J. Z., \& Hu, J. Y. 1998, A\&AS, 132, 173

Loidl, R., Lançon, A., \& Jorgensen, U. G. 2001, A\&A, 371, 1065

Martin, D. C., Fanson, J., Schiminovich, D., et al. 2005, ApJ, 619, L1

Massey, P. 2002, ApJS, 141, 122

Matsuura, M., Wood, P. R., Sloan, G. C., et al. 2006, MNRAS, 371, 415

Matsuura, M., Barlow, M. J., Zijlstra, A. A., et al. 2009, MNRAS, 396, 918

Meixner, M., Gordon, K. D., Indebetouw, R., et al. 2006, AJ, 132, 2288

Mokiem, M. R., de Koter, A., Puls, J., et al. 2005, A\&A, 441, 711

Murakami, H., Baba, H., Barthel, P., et al. 2007, PASJ, 59, S369

Onaka, T., De Jong, T., \& Willems, F. J. 1989, A\&A, 218, 1690

Onaka, T., Matsuhara, H., Wada, T., et al. 2007, PASJ, 59, S401

Pitman, K. M., Speck, A. K., \& Hofmeister, A. M. 2006, MNRAS, 371, 1744

Posch, T., Kerschbaum, F., Mutschke, H., et al. 1999, A\&A, 352, 609

Puls, J., Markova, N., Scuderi, S., et al. 2006, A\&A, 454, 625

Rieke, G. H., Young, E. T., Engelbracht, C. W., et al. 2004, ApJS, 154, 25

Rosario, M. J., Raveendran, A. V., \& Mekkaden, M. V. 2007, A\&A, 474, 41

Skrutskie, M. F., Cutri, R. M., Stienning, R., et al. 2006, ApJ, 131, 1163

Sloan, G. C., Levan, P. D., \& Little-Marenin, I. R. 1996, ApJ, 463, 310

Sloan, G. C., Kraemer, K. E., Price, S. D., et al. 2003a, ApJS, 147, 379

Sloan, G. C., Kraemer, K. E., Goebel, J. H., et al. 2003b, ApJS, 594, 483

Speck, A. K., Barlow, M. J., \& Skinner, C. J. 1997, MNRAS, 288, 431

Speck, A. K., Corman, A. B., Wakeman, K., et al. 2009, ApJ, 691, 1202

Srinivasan, S., Meixner, M., Leitherer, C., et al. 2009, AJ, 137, 4810

Stephenson, C. B. 1984, Publications of the Warner \& Swasey Observatory, 3, 1

Stephenson, C. B. 1990, AJ, 100, 569

Szczerba, R., Siódmiak, N., Stasińska, G., et al. 2007, A\&A, 469, 799

Sylvester, R. J., Kemper, C., Barlow, M. J., et al. 1999, A\&A, 352, 587

Takita, S., Kataza, H., Kitamura, Y., et al. 2010, A\&A, submitted

Tanabé, T., Sakon, I., Cohen, M., et al. 2008, PASJ, 60, 375

Van Den Ancker, M. E., The, P. S., Tjin A Djie, H. R. E., et al. 1997, A\&A, 324, 33

Van Den Ancker, M. E., de Winter, D., \& Tjin A Djie, H. R. E. 1998, A\&A, 330, 145

van der Hucht, K. A. 2001, NewAR, 45, 135

van der Veen, W. E. C. J., \& Habing, H. J. 1988, A\&A, 194, 125

van Leeuwen, F. 2007, Hipparcos, the new reduction of the raw data, ASSL (Dordrecht: Springer), 350

Voges, W., Aschenbach, B., Boller, Th., et al. 1996, The ROSAT All-Sky Survey Bright Source Catalogue

Waters, L. B. F. M., Coté, J., \& Lamers, H. J. G. L. M. 1987, A\&A, 185, 206

Weingartner, J. C., \& Draine, B. T. 2001, ApJ, 548, 296

Wenger, M., Ochsenbein, F., Egret, D., et al. 2000, A\&AS, 143, 9

Whitney, B. A., Wood, K., Bjorkman, J. E., et al. 2003, ApJ, 598, 1079

Wright, A. E., \& Barlow, M. J. 1975, MNRAS, 170, 41

York, D. G., Adelman, J., Anderson, J. E., et al. 2000, AJ, 120, 1579

Zhang, P., Chen, P. S., \& He, J. H. 2004, New Astron., 9, 509

Zhang, P., Chen, P. S., \& Yang, H. T. 2005, New Astron., 10, 325 